\title{
Poor quality evidence suggests that failure rates for atraumatic restorative treatment and conventional amalgam are similar
}

\author{
Abstracted from \\ Mickenautsch S, Yengopal V. \\ Failure rate of atraumatic restorative treatment using high-viscosity glass-ionomer cement \\ compared to that of conventional amalgam restorative treatment in primary and permanent teeth: \\ a systematic review update. Journal of Minimum Intervention in Dentistry 2012 5: 63-124 \\ Address for correspondence Steffen Mickenautsch, Department of Community Dentistry, Faculty of Health Sciences, \\ University of the Witwatersrand - 7 York Rd, Parktown/Johannesburg 2193, South Africa. neem@global.co.za.
}

\section{Question: This systematic review update seeks to answer the question whether, in patients with carious cavities of any class in primary and permanent teeth, ART restorations with high- viscosity GIC have a higher failure rate than amalgam restorations placed with conventional rotary instruments, in tooth cavities of the same size, type of dentition and follow-up period after one or more years.}

Data sources The Medline, Cochrane CENTRAL, Biomed Central, Database of Open Access Journals (DOAJ), OpenJ-Gate, Bibliografia Brasileira de Odontologia (BBO), LILACS, IndMed, Sabinet, Scielo, Scirus (Medicine), OpenSIGLE and Google Scholar databases were searched. Hand searching was performed for journals not indexed in the databases. References of included trials were checked.

Study selection Prospective clinical trials with test and control groups with a follow up of at least one year were included.

Data extraction and synthesis Data abstraction was conducted independently and clinical and methodologically homogeneous data were pooled using a fixed-effects model.

Results Eighteen trials were included. From these 32 individual dichotomous datasets were extracted and analysed. The majority of the results show no differences between both types of intervention. A high risk of selection-, performance-, detection- and attrition bias was identified. Existing research gaps are mainly due to lack of trials and small sample size.

Conclusions The current evidence indicates that the failure rate of high-viscosity GIC/ART restorations is not higher than, but similar to that of conventional amalgam fillings after periods longer than one year. These results are in line with the conclusions drawn during the original systematic review. There is a high risk that these results are affected by bias, and thus confirmation by further trials with suitably high numbers of participants is needed.

\section{Commentary}

In the mid 1980s atraumatic restorative treatment (ART) was developed as a means to deliver restorative treatment to rural communities without access to western-style dental clinics. ${ }^{1}$ It involves the removal of caries using hand instruments and restoration of the cavities with, primarily, glass-ionomer cements, (GIC) though the original descriptions of ART include the use of any adhesive material in combination with hand excavation. GICs come in both low and high viscosities, the latter being developed after the introduction of ART to make them more wear resistant.

To my knowledge there have been three systematic reviews comparing ART to conventional treatment, ${ }^{2-4}$ one of which was published by these authors. This systematic review asked: for deciduous and permanent teeth with any class of cavity, how do ART restorations using high viscosity GIC compare to conventional treatment using amalgam in terms of failure rate after at least one year?

It seems to be customary in the ART literature to compare ART with conventional treatment involving only amalgam as the restorative material. From an experimental point of view this is problematic because there are two variables. Either or both of these could account for differences in effectiveness between the two groups the means for removing caries (hand versus rotary instruments) and the means for restoring the cavity (GIC versus amalgam).

A further concern that this review question raises (and which is a reflection of the ART research literature) is whether conventional treatment is in fact the most appropriate comparison for the population ART was originally intended for. As described above, ART was developed for communities without access to electricity and water and for whom, presumably, the only alternative would be tooth extraction as a result of pain and/or sepsis. The assumption that deciduous teeth need to be restored is only now being tested in the FiCTION randomised controlled trial. ${ }^{5}$ As sacrilegious as it may sound, it is possible that deciduous teeth restored with either ART or conventional treatment fare no better than teeth left unrestored.

If instead we want to assess ART compared to other restorative options for patients with access to conventional treatment, ought we to be considering it in relation to those as well? Current restorative options include the Hall technique, conventional stainless steel crowns, conventional treatment with composite, ART with composite and more besides. Trials and systematic reviews of the effectiveness of ART in this population must, logically, include these alternatives.

Putting these concerns aside, the review authors searched a large number of relevant databases, including the BBO and LILACS 
databases from Latin America and the Caribbean. They didn't search any of the Chinese medical databases that have English interfaces. ${ }^{6}$ Searching these databases for ART does result in a number of potentially relevant trials of a reasonable size. However, the authors did identify eight Chinese trials probably through Google Scholar. Unfortunately these had not been translated when this report was written. * This is a shame as the trials may have added valuable new data and thus the review is at risk of a language bias.

The search strategies are limited and, interestingly, the PubMed search for this review included 'Art' $[\mathrm{MeSH}]$ that resulted in 19,766 hits. This MeSH term is reserved for the kind of art that involves painting, engraving and sculpture so I am unclear why it was used.

Randomisation reduces the risk of selection bias compared to other study designs. Studies that do not use adequate randomisation procedures tend to overestimate the effectiveness of the test intervention. ${ }^{7}$ The inclusion criteria here for studies was 'prospective, clinical controlled trials'. Thus non-random trials could be eligible. Since in these studies the test intervention will be ART most of the time, given the previous statement, it is likely that they will overestimate its effect. It would therefore be useful to know what proportion of the included trials were randomised. This information I could not find.

The authors planned to assess the selection bias risk using a method unfamiliar to me. I could not find a reference that might have helped me understand it. The Cochrane handbook - available online - offers sound guidance on how to assess the various bias risks involved in conducting RCTs and has a straightforward tool to assist reviewers in doing this. ${ }^{8}$

This method was complex and seems confused. For example, when assessing 'adequate random sequence generation method reported' it does not explain what adequate is but instead states that 'inadequate' includes cluster randomisation, randomisation of teeth, split-mouth study design and generation of the random sequence before patient recruitment. These are not ways of generating an allocation sequence. By sequence generation we mean the way in which the allocation of a patient to one intervention or the other is actually arrived at. This can be done, for example, by deciding that every person who arrives on a Tuesday receives intervention A and everyone arriving on a Wednesday receives intervention B. This is clearly not random. Adequate randomisation may use simple means such as rolling a dice (eg patients with even numbers get $\mathrm{A}$, those with odd numbers get $\mathrm{B}$ ) but more often by using a published list of random numbers or a list of random assignments generated by a computer. ${ }^{8}$

There are a number of tables in this review but none includes a summary of the included trials. There are tables with summaries of the data sets that the authors extracted, though, but these are complex and difficult to make sense of.

Where there was sufficient clinical homogeneity the authors have combined different data sets from the various studies in a metaanalysis, eg 'single surface restorations placed in primary teeth and evaluated according to the ART criteria after 1 year'. Six such analyses were conducted. I was unclear how useful a grouping entitled 'restorations evaluated according to USPHS criteria after 1 year' is, given that it doesn't specify whether the teeth were deciduous or permanent, nor which class these restorations were.

This review, along with the others, concludes that there is no statistical difference in the longevity of ART and amalgam for most situations, though this and two of the three previous reviews all concluded that the trials they considered were of low quality. Thus we must be wary about concluding that ART does perform as well as conventional treatment with amalgam. Furthermore, the number of trials conducted on permanent teeth appears to be small and there may not even be a single truly randomised controlled trial in these teeth. Thus any conclusions we make regarding ART should bear in mind that the research is primarily in relation to primary teeth, which clearly have a shorter natural lifespan than permanent teeth.

This paper is complicated to read and this appears to obfuscate some methodological problems as well as making its message unclear for the average reader. The PRISMA guidelines have been around for some time and were created to aid in the clear reporting of systematic reviews. ${ }^{9}$ I believe this review would have been more useful to clinicians and policy makers if these guidelines had been followed.

For the large proportion of the world's population without access to 'conventional' clinics, we still need to understand more about the need for restorative treatment per se, and the suitability of ART or something similar for this purpose in both deciduous and permanent teeth.

Dominic Hurst

Queen Mary University of London, Barts \& The London School of Medicine and Dentistry, Institute of Dentistry, $U K$.

1. Frencken JE. Evolution of the ART approach: highlights and achievements. J App/ Oral Sci. 2009; 17: 78-83.

2. Frencken JE, Van 't Hof MA, Van Amerongen WE, Holmgren CJ. Effectiveness of single-surface ART restorations in the permanent dentition: a meta-analysis. J Dent Res. 2004; 83: 120-123.

3. Mickenautsch S, Yengopal V, Banerjee A. Atraumatic restorative treatment versus amalgam restoration longevity: a systematic review. Clin Oral Investig. 2010; 14: 233-240.

4. Pettar M, Zhao I, Wu T, Memetimin N, Liu Z. Atraumatic Restorative Treatment versus Conventional Restorative Treatment for Childhood Caries: A Systematic Review. Chinese Journal of Evidence-Based Medicine. 2011; 11: 413-418.

5. National Institute for Health Research (NIHR). NIHR Health Technology Assessment Programme: FiCTION - Filling Children's Teeth: Indicated Or Not?. 2012 [cited 2012 22/04/2012]; Available from: http://www.hta.ac.uk/1783.

6. Xia J, Wright J, Adams CE. Five large Chinese biomedical bibliographic databases: accessibility and coverage. Health Info Libr J. 2008; 25: 55-61. Epub 2008/02/07.

7. Kunz R, Vist G, Oxman AD. Randomisation to protect against selection bias in healthcare trials. Cochrane Database Syst Rev. 2007; (2): MR000012. Epub 2007/04/20.

8. Higgins J, Green SP. Cochrane handbook for systematic reviews of interventions. Oxford: Wiley-Blackwell; 2008.

9. Liberati A, Altman DG, Tetzlaff I, Mulrow C, Gotzsche PC, loannidis IP, et al. The PRISMA statement for reporting systematic reviews and meta-analyses of studies that evaluate health care interventions: explanation and elaboration. PLoS Med. 2009; 6: e1000100. Epub 2009/07/22.

\section{* Editors Note}

An update to the Mickenautsch and Yengopal review is available for the Journal of Minimum Intervention in Dentistry which now includes data from the Chinese studies. This has not however affected the main conclusion from the review

Evidence-Based Dentistry (2012) 13, 46-47. doi:10.1038/sj.ebd.6400858 